\title{
BASIC APPROACH IN THE MICROSCOPIC THEORY OF METALS
}

\author{
V. B. Solovyan \\ Institute for Condensed Matter Physics of the Ukrainian National Academy of Sciences \\ 1 Svientsitsky Str., UA-290011, Lviv, Ukraine
}

(Received 31 January 1997)

\begin{abstract}
A scheme of the construction of the many-body microscopic metallic state theory which is based on the two main principles, is proposed. The first principle consists in the use of the optimal representation basic set with orths close to one-particle quantum states in different electron subsystems of metal. The second one consists in the choice of the corresponding reference system as a statistical basis of description. The developed approach allows to describe different functional subsystems of electrons in metal as well as the effects of their mutual interaction with the correct allowance for the many-particle correlation effects.
\end{abstract}

Key words: Basic approach, functional electron subsystems, ortogonalization, statistical operator reduction.

PACS number(s): $71 \cdot 10 .+\mathrm{x}$

\section{INTRODUCTION}

A transition from one-particle semifenomenological description to the many-particle microscopic one forms the principal peculiarity of the modern metallic theory evolution. The coexistence of two electron subsystems - localized and collectivised - determines the metallic state specifity. It follows from the main problem of the microscopic metal theory - the adequate description of both electron subsystems with the allowance for their mutual interacting effects.

It is well known that the precise description of both isolated electron subsystems - localized and collectivised - can be done with the help of quantum chemistry and the electron liquid methods, correspondingly. There are two main approaches to the allowance for interaction between these two different electron subsystems in the present-day theory of non-transition metals. The first approach is based on the pseudopotential conception (or model potential) $[1,2]$. The second one - on the reduction of statistical operator in the exact electron-nuclear model by the averaging over the localized electron states [3]. In such metals the separation of electrons into two different subsystems is physically substantiated by the existence of deep energy levels of localized electrons and compact ionic cores. It follows that the localized electron states are determinated clearly. Pseudopotentials (model potentials) or the effective potentials of electron-ion interaction are too weak here which makes it possible to use of perturbation theory to take into account mutual interaction between two electron subsystems [4]. However, the pseudopotential description of such simplest metallic systems is not adequate in the whole region of external parameters (see [5]).

The success of the electron-ion model in the theory of nontransition metals is due to the fact that the electrons of ionic internal shells play a passive role in many physical processes.

An essentially different pattern arises in transition metals where the localized $d$-electrons of external shell play the active role in physical processes. In this case the separation itself of the electrons into localized and delocalized loses an absolute significance, so does the electron-ion model conception. A strong gibridization between conductive and $d$-electrons is caused by a small value of excitation energies. Its rigorous account cannot be done in terms of pseudopotential or effective potentials methods.

A particular interest represents the situation when the ionic core changes itself with the variation of external parameters. It takes place for the systems with changeable valency where the portion of $f$-electrons becomes delocalized or the $f$-state character itself changes sharply (the collapse phenomenon). For the "anomalous" $f$-systems the "localize - delocalize dualism" of electron states comes forth with particular strength [5]. Above we have considered pure metals. The important field of metallic state theory forms metals with impurities. The microscopic description of such objects becomes more difficult as far as we have no apriory information about the character of the electron states localized in the impurities region. The presented examples certify that the electron-ion model conception in its modern version is far from universality.

In the present paper a general scheme of constructing the many-particle microscopic metal state theory in terms of renormalized perturbation theory is proposed. It is well known that precise calculations of the physical system characteristics are invariant over the representation basic-set. However, for the approximate calculations (which are the only possible ones for many-particle systems) it is not the case - for different basis we obtain different results. It follows from the essentiality of the use of such a basis representation, having basic functions close to one-particle wave functions of electron states of a physical system (optimal basic-set). This provides for the speedy convergentive perturbation theory. In the case of metallic state such basic-set must include functions close to one-particle wave functions in functionally different 
isolated electron subsystems.

A rigorous account of the correlation effects in the isolated electron subsystems, especially what concerns their mutual influence in terms of a basis-set, is impossible in principle. This fact moves forward the problem of their correct account with the help of an adequate formulation of perturbation theory. A successive solution of this problem fully depends on zero approximation which in its turn depends on adequate reference system (statistical basis). The suggested approach is based on the use of optimal basis of representation and on the choice of adequate reference system.

\section{MODEL AND BASIS OF REPRESENTATION}

Let us consider the electron-nuclei model of the electroneutral metallic system which consists of $\mathrm{M}$ sorts of nuclei with the charge $Q_{a} e(1 \leq a \leq M)$ and $N_{e}$ electrons in the volume $\mathrm{V}$ in the thermodynamic limit $N_{e}, V \rightarrow \infty, N_{e} V^{-1}=$ const. In order to simplify the calculations we shall use the adiabatic approximation for the ionic subsystem, describing the ions in coordinate representation. With the same aim we shall neglect the relativistic effects too. In that case the Hamiltonian of the considered model in coordinate representation takes the form of

$$
\begin{aligned}
\hat{H} & =\hat{H}_{\text {nucl }}(\mathbf{R})-\sum_{j=1}^{N_{e}} \frac{\hbar^{2} \nabla^{2}}{2 m} \\
& -\sum_{a=1}^{M} Q_{a} e^{2} \sum_{i=1}^{N_{a}} \sum_{j=1}^{N_{e}}\left|\mathbf{r}_{j}-\mathbf{R}_{i}^{a}\right|^{-1}+\sum_{i \neq j=1}^{N_{e}} e^{2}\left|\mathbf{r}_{j}-\mathbf{r}_{i}\right|^{-1} .
\end{aligned}
$$

Here, $\hat{H}_{n u c l}(\mathbf{R})$ is the Hamiltonian of nuclei subsystem; $\mathbf{R}_{j}^{a}$ is the radius-vector of the $j$-th nuclei of sort $a ; m$ and $e$ is the mass and the charge of the electron. To carry out the construction of this model, we shall assume proceeding from general physical considerations, that there may exist $n$ functional different subsystems of localized electrons $(n \geq M)$ and a subsystem of collectivised electrons in the model. Here, the condition of properly defined localized states cannot be satisfied ( small excitation energies or a large radius of the corresponding localized states may take place). Such localized electrons subsystems can conform to the electrons of ionic cores, $d$ - and $f$-electrons, to the electrons localized in the impurity region, vacancy, etc.

We shall construct the optimal basis of functions $\left\{\Psi_{\sigma}(\mathbf{r})\right\}$ from the plane wave basis $\left\{\varphi_{\mathbf{k}}(\mathbf{r})\right\} \equiv$ $\left\{V^{-1 / 2} \exp (i \mathbf{k r})\right\}$ and $n$ subspaces of the atomic-like localized functions $\left\{\Phi_{\beta_{a}}\left(\mathbf{r}-\mathbf{R}_{j}^{a}\right)\right\}$. Here $\beta_{a}$ is a set of quantum numbers of state and the wave-function is centered on the $j$-th nuclei of the sort $a$. The functions $\Phi_{\beta_{a}}\left(\mathbf{r}-\mathbf{R}_{j}^{a}\right)$ may be considered as well known, or be determined simultaneously. We shall consider that the overlap integrals

$$
S_{\alpha_{a} \beta_{b}}\left(\mathbf{R}_{i}^{a}-\mathbf{R}_{j}^{b}\right)=\left\langle\Phi_{\alpha_{a}}\left(\mathbf{r}-\mathbf{R}_{i}^{a}\right) \mid \Phi_{\beta_{b}}\left(\mathbf{r}-\mathbf{R}_{j}^{b}\right)\right\rangle
$$

are equal to $\delta_{\alpha_{a}, \beta_{b}}$ at $a=b, i=j$, and their values in the other cases are much smaller in magnitude in comparison with the unit. It serves as a justification for the approximate orthogonalization procedure for the functions of these subspaces on the basis of forming their linear combinations

$$
\Psi_{\alpha_{a}}^{a, i}(\mathbf{r})=\sum_{b, \beta_{b}, j} U_{\beta_{b}, \alpha_{a}}^{b, a}(i, j) \Phi_{\beta_{b}}\left(\mathbf{r}-\mathbf{R}_{j}^{b}\right)
$$

The transformation coefficients $U_{\beta_{b}, \alpha_{a}}^{b, a}(i, j)$ are presented in the form of expansion in terms of overlap integrals. In order to follow the given accuracy further we must stick to the same approximation as for $U_{\beta_{b}, \alpha_{a}}^{b, a}(i, j)$. Let us construct the basis of functions in the form of

$$
\begin{aligned}
\left\{\Psi_{\sigma}(\mathbf{r})\right\}= & \left\{\Psi_{\sigma_{1}}(\mathbf{r})\right\} \oplus\left\{\Psi_{\sigma_{2}}(\mathbf{r})\right\} \oplus \cdots \\
& \oplus\left\{\Psi_{\sigma_{n}}(\mathbf{r})\right\} \oplus\left\{\Psi_{\sigma_{n+1}}(\mathbf{r})\right\}
\end{aligned}
$$

where $n$ subspaces $\left\{\Psi_{\sigma_{s}}(\mathbf{r})\right\}$ represent the whole functions set (2.3) and the subspace $\left\{\Psi_{\sigma_{n+1}}(\mathbf{r})\right\} \equiv\left\{\Psi_{\mathbf{k}}(\mathbf{r})\right\}$ is constructed due to methods of papers $[7,8]$ on the base of $\left\{\Psi_{\sigma_{s}}(\mathbf{r})\right\} \quad(1 \leq s \leq n)$ and a plane waves basis $\left\{\varphi_{\mathbf{k}}(\mathbf{r})\right\}$. It must be noted here that the wave-vector $\mathbf{k}$ takes on all values, allowed by Born-Carman's boundary conditions, but for the set $\left\{\mathbf{k}_{s}\right\}$, which contains as many vectors, as many functions $\Psi_{\sigma_{s}}(\mathbf{r})$ were introduced into the basis $\left\{\Psi_{\sigma}(\mathbf{r})\right\}$. Functions $\Psi_{\sigma}(\mathbf{r})$ form a complete and orthonormal system (basis) so far as

$$
\begin{aligned}
& \sum_{\sigma} \Psi_{\sigma}^{*}\left(\mathbf{r}_{1}\right) \Psi_{\sigma}\left(\mathbf{r}_{2}\right)=\delta\left(\mathbf{r}_{1}-\mathbf{r}_{2}\right) \\
& \left\langle\Psi_{\sigma^{\prime}} \mid \Psi_{\sigma^{\prime \prime}}\right\rangle=\delta_{\sigma^{\prime}, \sigma^{\prime \prime}}
\end{aligned}
$$

and $\delta_{a, b}$ - the Kroneker symbol, $\delta(a-b)$ - the Dirac delta-function $\left(\sigma \equiv\left\{\sigma_{1}, \sigma_{2}, \ldots, \sigma_{n}, \mathbf{k}\right\}\right)$. In the case when nuclei form a crystal lattice in order to avoid the degeneration it is sensible to introduce into basis (2.4) instead of functions $\left\{\Psi_{\sigma_{s}}(\mathbf{r})\right\}$ the corresponding subspace of Bloch functions.

The functions $\Psi_{\sigma}(\mathbf{r})$ arise as a result of the canonical transformation of the plane wave basis and proceeds by the unitary operator $\hat{U}$ :

$$
\Psi_{\sigma}(\mathbf{r})=\hat{U} \varphi_{\mathbf{q}}(\mathbf{r})= \begin{cases}\Psi_{\sigma_{s}}(\mathbf{r}) & \mathbf{q} \in\left\{\mathbf{k}_{s}\right\} \\ \Psi_{\mathbf{k}}(\mathbf{r}) & \mathbf{q} \notin\left\{\mathbf{k}_{s}\right\}\end{cases}
$$

They are eigenfunctions of a linear Hermitian operator $\hat{\Lambda}=\hat{U} \hat{T} \hat{U}^{+}$and obviously the spectrum of the operator $\hat{\Lambda}$ coincides with the spectrum of the kinetic energy operator $\hat{T}=-\hbar^{2} \nabla^{2} / 2 m$.

We transform the Hamiltonian (2.1) from coordinate representation to another one using basis (2.4): 


$$
\begin{aligned}
& \hat{H}=\hat{H}_{n u c l}(\mathbf{R})+\sum_{\sigma_{1}, \sigma_{2}} \sum_{s} b_{\sigma_{1}, \sigma_{2}} a_{\sigma_{1}, s}^{+} a_{\sigma_{2}, s} \\
& +\frac{1}{2 V} \sum_{\sigma_{1}, \ldots, \sigma_{4}} \sum_{s_{1}, s_{2}} V_{\sigma_{1}, \sigma_{2}, \sigma_{3}, \sigma_{4}} a_{\sigma_{1}, s_{1}}^{+} a_{\sigma_{2}, s_{2}}^{+} a_{\sigma_{3}, s_{2}} a_{\sigma_{4}, s_{1}}
\end{aligned}
$$

where the following symbols for matrix elements are used:

$$
\begin{aligned}
& b_{\sigma_{1}, \sigma_{2}}=T_{\sigma_{1}, \sigma_{2}}-V^{-1} \sum_{a=1}^{M} Q_{a} \sum_{\mathbf{q}} V_{q} S_{-\mathbf{q}}^{a} R_{\sigma_{1}, \sigma_{2}}(\mathbf{q}), \\
& V_{\sigma_{1}, \sigma_{2}, \sigma_{3}, \sigma_{4}}=V^{-1} \sum_{\mathbf{q}} R_{\sigma_{1}, \sigma_{4}}(\mathbf{q}) R_{\sigma_{2}, \sigma_{3}}(-\mathbf{q}), \\
& R_{\sigma_{1}, \sigma_{2}}(\mathbf{q})=V^{1 / 2}\left\langle\Psi_{\sigma_{1}} \mid \varphi_{\mathbf{q}} \Psi_{\sigma_{2}}\right\rangle \\
& T_{\sigma_{1}, \sigma_{2}}=\left\langle\Psi_{\sigma_{1}}|\hat{T}| \Psi_{\sigma_{2}}\right\rangle, \quad S_{\mathbf{q}}^{a}=\sum_{j=1}^{N_{a}} \exp \left(i \mathbf{q} \mathbf{R}_{j}^{a}\right)
\end{aligned}
$$

Here, $V_{q} \equiv 4 \pi e^{2} q^{-2}$ and operators $a_{\sigma, s}$ are up to the basic functions $(s= \pm 1 / 2$ is spin variable). The use of the basis $\left\{\Psi_{\sigma}\right\}$ gives a possibility for possessing description of all electron subsystems.

\section{REFERENCE SYSTEM AND RENORMALIZED PERTURBATION THEORY}

The ultimate aim of this consideration is the calculation of partition function by means of averaging over electron variables in grand canonical ensemble

$$
Z(\mu)=S p \hat{\rho}=S p\{\exp [-\beta(\hat{H}-\mu \hat{N})]\}=\exp [-\beta \Omega(\mu)]
$$

where $\mu$ is the chemical potential variable, $N=$ $\sum_{\sigma, s} a_{\sigma, s}^{+} a_{\sigma, s}$ is the operator of total number of electrons in the system, $\Omega(\mu)$ is the grand thermodynamic potential. There are some electron subsystems among the considered $(n+1)$ ones, which are sufficiently isolated and have a weak influence on other subsystems, which play an active role in physical processes. A subsystem of localized electrons in nontransition metals, a subsystem of core electrons in $d$ - or $f$-metals, etc. can serve as examples of such a sufficiently isolated system. This substantiates the reduced description of model (2.7) and the calculation of its partition function in step-by-step form. It demands the calculation of partitial functions just over the electron states of some $L$ subsystems (over $L$ subspaces of the basis (2.4))

$$
\begin{aligned}
& Z_{L}(\mu)=S p_{1, \ldots, L}\{\exp [-\beta(\hat{H}-\mu \hat{N})]\}=\hat{P}_{L} \\
& L \leq n+1
\end{aligned}
$$

The partial underintegrated partitial function $Z_{L}(\mu)$ determines the effective statistical operator $\hat{P}_{L}$ for other $(n+1-L)$ subsystems or the effective Hamiltonian of a reduced model which deals with the mentioned $(n+1-L)$ electron subsystem.In particular, when only one subsystem is of special interest a reduction of the statistical operator over all subsystems, except that of $(L=n)$, can be done. At $L=n+1$ formulae (3.2) defines a complete partition function of the whole model. Consequently by definition

$$
\begin{aligned}
Z(\mu) & =S p_{n+1} Z_{n}(\mu) \\
& =S p_{n, n+1} Z_{n-1}(\mu)=\ldots=S p_{1, \ldots, n+1} \hat{\rho} .
\end{aligned}
$$

In some sense the procedure proposed here is the quantum analogue of the step-by-step integration method used for the calculation of partition function of the classic Ising model (see [9]). We shall explain this by giving examples. For the pure nontransition metal the reduction over the states of the localized electron subsystem results in the microscopic electron-ion model. In the case of pure transition metal the reduction over the core electron states brings to the model which describes subsystems of $d$ - and $f$-electrons in the effective field of ions. In the presence of the hydrogen impurities in metal the mentioned reduction over the states of all electron subsystems of the pure metal gives the effective Hamiltonian for the electron subsystem in the field of protons and metallic "atoms". Statistical averaging over the states of the collectivised electron subsystem in the nontransition metals gives the off-beat quantum ionic model of metal. A statistical reduction procedure was used in the papers $[3,10]$ for the transition from the exact electronnuclei model of the nontransition metal to an approximate electron-ion model.

For the $Z(\mu)$ calculation we use the renormalized perturbation theory, proceeding from the statistical operator to the interaction presentation on the basis of some operator $\hat{H}_{0}^{L}$. As a result of this procedure we obtain

$$
\begin{aligned}
& Z_{L}(\mu)=Z_{L}^{0}(\mu)\left\langle\hat{S}_{L}\right\rangle_{0} \\
& Z_{L}^{0}(\mu)=S p_{1, \ldots, L} e^{-\beta \hat{H}_{0}^{L}} \\
& \hat{S}_{L}=T \exp \left\{-\int_{0}^{\beta} d \beta^{\prime}\left[\hat{H}\left(\beta^{\prime}\right)-\hat{H}_{0}^{L}\left(\beta^{\prime}\right)-\mu \hat{N}\left(\beta^{\prime}\right)\right]\right\}
\end{aligned}
$$

Here $Z_{L}^{0}(\mu)$ is the partition function of the model with the Hamiltonian $\hat{H}_{0}^{L}$, the symbol $\langle\ldots\rangle_{0}$ stands for a statistical averaging over this model states, $T$ is the symbol of ordinary chronological ordering [11], and $\hat{H}\left(\beta^{\prime}\right), \hat{N}\left(\beta^{\prime}\right), \hat{H}_{0}\left(\beta^{\prime}\right)$ - corresponding operators in the interaction presentation. Both in papers [3, 10] and $[12,13]$ (devoted to the electron liquid model) in the capacity of the operator $\hat{H}_{0}^{L}$ the Hamiltonian of noninteracting particles (or quasiparticles) — the diagonal 
quadratic Hamiltonian of the type $\sum_{\sigma, s} \varepsilon_{\sigma} a_{\sigma, s}^{+} a_{\sigma, s}$ was used. Such a choice is justified in the case of a weak interaction both between electrons inside some subsystem and between different subsystems. In the general case we shall choose the nondiagonal operator in the capacity of operator $\hat{H}_{0}^{L}$

$$
\begin{aligned}
& \hat{H}_{0}^{L}=\sum_{\substack{\lambda_{1}, \lambda_{2} \\
s}}\left(\varepsilon_{\lambda_{1}, \lambda_{2}}-\mu \delta_{\lambda_{1}, \lambda_{2}}\right) a_{\lambda_{1}, s}^{+} a_{\lambda_{2}, s}, \\
& 1 \leq L \leq n+1,
\end{aligned}
$$

where $\lambda_{1}, \lambda_{2} \in\left\{\sigma_{1}, \ldots, \sigma_{L}\right\}$. The matrix elements $\varepsilon_{\lambda_{1}, \lambda_{2}}$ can be obtained by some approximation from the initial Hamiltonian, or they are considered as variational parameters which are obtained self-consistently in the process of solving the problem. Really the thermodynamic potential $\Omega(\mu)$ forms a functional on the unknowns $\varepsilon_{\lambda_{1}, \lambda_{2}}$. However, it is obvious that with the allowance of perturbation theory for all diagrams the series $\Omega(\mu)$ becomes invariant over the $\hat{H}_{0}^{L}$ choice and independent on $\varepsilon_{\lambda_{1}, \lambda_{2}}$ :

$$
\frac{\delta \Omega(\mu)}{\delta \varepsilon_{\lambda_{1}, \lambda_{2}}}=0
$$

For approximate calculations which are practically the only possible ones formula (3.6) becomes the extremum condition for thermodynamic potential and determines the system of equations for the unknown matrix elements $\varepsilon_{\lambda_{1}, \lambda_{2}}$.

The nondiagonal terms of the operator $\hat{H}_{0}^{L}$ take into account the gibridization effects both between different basic states inside one subsystem and between different electron subsystems. This becomes sufficiently important in the case of $d$ - and $f$-metals, the presence of impurity, etc. However, the calculation of $\left\langle\hat{S}_{L}\right\rangle_{0}$ on the use of interaction presentation on the basis of nondiagonal operator (3.5) becomes too difficult. In order to avoid this difficulty we diagonalize $\hat{H}_{0}^{L}$ by transition from the operators $a_{\lambda, s}$ to "quasiparticle" Fermi operators $c_{\nu, s}$ on the base of canonical transformation

$$
\begin{aligned}
a_{\lambda, s} & =\sum_{\nu} \zeta_{\lambda, \nu} c_{\nu, s}, \\
c_{\nu, s} & =\sum_{\lambda} \xi_{\nu, \lambda} a_{\lambda, s}, \quad\{\nu\} \equiv\{\lambda\} .
\end{aligned}
$$

The transformation coefficients $\zeta_{\lambda, \nu}$ determine the oneparticle spectrum of the model system described by Hamiltonian $\hat{H}_{0}^{L}$ :

$$
E_{\nu}=\sum_{\lambda_{1}, \lambda_{2}}\left[\varepsilon_{\lambda_{1}, \lambda_{2}}-\mu \delta_{\lambda_{1}, \lambda_{2}}\right] \zeta_{\lambda_{1}, \nu}^{*} \zeta_{\lambda_{2}, \nu}
$$

And for the determination of the coefficients $\zeta_{\lambda, \nu}$ we obtain the following system of equations:

$$
\begin{aligned}
& \sum_{\lambda_{1}, \lambda_{2}}\left[\varepsilon_{\lambda_{1}, \lambda_{2}}-\mu \delta_{\lambda_{1}, \lambda_{2}}\right] \zeta_{\lambda_{1}, \nu_{1}}^{*} \zeta_{\lambda_{2}, \nu_{2}}=0 \text { at } \nu_{1} \neq \nu_{2} \\
& \sum_{\nu} \zeta_{\lambda_{2}, \nu}^{*} \zeta_{\lambda_{1}, \nu}=\delta_{\lambda_{1}, \lambda_{2}}
\end{aligned}
$$

In terms of quasiparticles the Hamiltonian $\hat{H}_{0}^{L}$ takes the form of:

$$
\hat{H}_{0}^{L}=\sum_{\nu, s} E_{\nu} c_{\nu, s}^{+} c_{\nu, s}
$$

which is in conformity with the unitary transformation of subspace $\left\{\Psi_{\sigma_{1}}\right\} \oplus\left\{\Psi_{\sigma_{2}}\right\}+\ldots+\left\{\Psi_{\sigma_{L}}\right\}$ of basis (2.4). Really, the electron field operator projection on this subspace can be represented both in $\Psi_{\lambda}$ and is some new functions $\tilde{\Psi}_{\nu}(\mathbf{r})$ which correspond to the operators $c_{\nu, s}$ :

$$
\hat{\Psi}^{(\lambda)}(\mathbf{r})=\sum_{\lambda} a_{\lambda, s} \Psi_{\lambda}(\mathbf{r})=\sum_{\nu} c_{\nu, s} \tilde{\Psi}_{\nu}(\mathbf{r})
$$

With the allowance for orthogonality of functions, both $\Psi_{\lambda}(\mathbf{r})$ and $\tilde{\Psi}_{\nu}(\mathbf{r})$, we obtain the following transformation rules

$$
\begin{aligned}
& \tilde{\Psi}_{\nu}(\mathbf{r})=\sum_{\lambda} \zeta_{\lambda, \nu} \Psi_{\lambda}(\mathbf{r}) \\
& \Psi_{\lambda}(\mathbf{r})=\sum_{\nu} \xi_{\nu, \lambda} \tilde{\Psi}_{\nu}(\mathbf{r})
\end{aligned}
$$

Thus, the transformation from the operators $a_{\lambda, s}$ to $c_{\nu, s}$ means the substitution of the initial basis (2.4) with a new basis

$$
\begin{gathered}
\left\{\tilde{\Psi}_{\sigma}\right\}=\left\{\tilde{\Psi}_{\nu_{1}}\right\} \oplus\left\{\tilde{\Psi}_{\nu_{2}}\right\} \oplus \ldots \oplus\left\{\tilde{\Psi}_{\nu_{L}}\right\} \oplus \\
\oplus\left\{\Psi_{\lambda_{L+1}}\right\} \oplus \ldots \oplus\left\{\Psi_{\lambda_{n}}\right\} \oplus\left\{\Psi_{\mathbf{k}}\right\}
\end{gathered}
$$

Obviously, the transformation from $\left\{\Psi_{\sigma}\right\}$ to $\left\{\tilde{\Psi}_{\sigma}\right\}-$ basis can be presented in the form of

$$
\tilde{\Psi}_{\sigma}(\mathbf{r})=\hat{\zeta} \Psi_{\sigma}(\mathbf{r})
$$

Taking into account the eigenvalue equation for the operator $\hat{\Lambda}$ one can find that the basis $\left\{\tilde{\Psi}_{\sigma}\right\}$ is formed by eigenfunctions of the linear Hermitian operator

$$
\hat{W}=\hat{\zeta} \hat{\Lambda} \hat{\zeta}^{+} \equiv \hat{\zeta} \hat{U} \hat{T} \hat{U}^{+} \hat{\zeta}^{+}
$$

The operator $\hat{\zeta}$ is the unitary operator which is defined by matrix clements $\zeta_{\lambda, \nu}$ on the subspace $\left\{\Psi_{\lambda_{1}}\right\} \oplus \ldots \oplus$ $\left\{\Psi_{\lambda_{L}}\right\}$ and on the additional complement $\left\{\Psi_{\lambda_{L+1}}\right\} \oplus \ldots \oplus$ 


\title{
V. B. SOLOVYAN
}

$\left\{\Psi_{\mathbf{k}}\right\}$ coinsides with the unit operator. Using the first formula (3.7) we transfer $\hat{S}_{L}$ (see (3.4)) from $a_{\lambda, s}$ to operators $c_{\nu, s}\left(\beta^{\prime}\right)$ on the basis of interaction representation with Hamiltonian (3.10). The choice of operator (3.5) makes it possible to speed up the convergence of a perturbation theory series. As a result of reduction (3.2) we obtain the effective statistical operator in the form of

$$
\hat{P}_{L}=\exp \left\{-\beta \hat{H}_{n u c l}(\mathbf{R})-\beta \Omega_{L}(\mu)-\beta \hat{H}_{e f}\left(a_{\sigma, s}\right)\right\},
$$

where $\Omega_{L}(\mu)$ is the grand thermodynamic potential of extracted $L$ subsystems, and $\hat{H}_{e f}\left(a_{\sigma, s}\right)$ is the effective Hamiltonian for all other electron subsystems of the physical system.

All well known methods of optimal basis construction $[7,8,14,15]$ are based on the purely mathematical principle: the plane wave basis and a subspace of localized functions are used; further their certain linear combinations form the basic functions which satisfy formal mathematical conditions of orthogonality and completeness (2.5). In this way the optimal basis construction, being purely mathematical, absolutely disregards the specific physical problems, in particular, the interaction between the different electron subsystems is neglected. Here it must be noted, that with the help of linear combinations of the initial basis functions (2.4) a continuum of mathematically equivalent optimal basis can be formed and their use brings to different physical results. In this situation some physical principle must be formulated which consists in the allowance for a specific physical system for an optimal basis choice. It consists in the imposition of an additional (except (2.5)) conditions set, which is to consider on the basis level the allowance for interaction between different electron subsystems, a strong convergence of renormalized perturbation theory series, etc. Mathematically it corresponds to zeroing the most important matrix elements which accounts for the interaction between subsystems, or in the general case it corresponds to the diagonalization of the Hamiltonian $\hat{H}_{0}^{L}$. This procedure is the logical generalization of the purely mathematical condition of mutual orthogonality for the basic functions (second formula in (2.4)), which in its turn is only a formal reason to consider the nondiagonal matrix elements to be small.

The basic approach suggested in this paper combines in the only possible way two important aspects: optimal representation basis and statistical basis (reference system). It provides for the speeding up of the convergency of the perturbation theory series with the allowance for various many-particle correlation effects.

It is my pleasant duty to express profound gratitude to Dr. M.Vavrukh, whose collaboration, scientific and moral support were of unquestionable significance in the process of writing the present paper.
[1] Heine V., Cohen M., Weaire D., Solid State Physics (Academic Press, New York-London, 1970).

[2] Harrison W. A., Pseudopotentials in the Theory of Metals (Benjamin, New York, 1966).

[3] Vavrukh M., Muliava Ya., J. Phys. Stud. 1, 257 (1996).

[4] Browman E. G., Kagan Yu., Kholas A., Zhurn. Eksp. Teor. Fiz. 61, No 1, 130 (1971).

[5] Vonsovskii S. V., Katsnelson M. I., Trefilov A. V., Fizika Metalov i Metalovedenije 76, No 3, 3 (1993).

[6] Bogoliubov N. N., The Quantum Statistic Lectures (Radyans'ka Shkola, Kiev, 1949).

[7] Solovyan V. B., Dopovidi Akad. Nauk (A), No 4, 68 (1987).

[8] Vavrukh M., Solovyan V., Ukr. Fiz. Zhurn. 36, No 4, 630 (1991).
[9] Yukhnovskii I. R., The Second Order Phase Transitions. The Collective Variables Method (Naukova Dumka, Kiev, 1985).

[10] Vavrukh M., Krokhmalskii T., Fiz. Metalov i Metalovedenije, 59, No 5, 864 (1985).

[11] Abrikosov A. A., Gor'kov L. P., Dzialoshinskii I. E. The Quantum Field Theory Methods in Statistical Physics (Fizmatgiz, Moskva, 1962).

[12] Vavrukh M., Krokhmalskii T., phys. stat. sol. (b) 168, 519 (1991).

[13] Vavrukh M., Vavrukh N., Low. Temp. Phys. 21, No 7, 572 (1995).

[14] Girardeau M. D., J. Math. Phys. 12, 165 (1971).

[15] Gurskii B. A., Gurskii Z. A., Ukr. Fiz. Zhurn. 21, No 10, 1603 (1976).

\section{БАЗИСНИЙ ПІДХІД У МІКРОСКОПІЧНІЙ ТЕОРІЇ МЕТАЛІВ}

\author{
В. Б. Солов'ян \\ Інститут фізики конденсованих систем Націоналъної академї наук Украйни \\ Украйна, UА-290011, Лъвів, вул. Свенціивкого, 1
}

\footnotetext{
Запропоновано схему побудови багаточастинкової мікроскопічної теорії металічного стану, яка грунтується на двох засадах. По-перше, використання оптимального базису представлення, орти якого близькі до одночастинкових станів в окремих підсистемах електронів металу. По-друге, вибір відповідної базисної системи як статистичного базису опису. Розвинутий підхід дозволяє адекватно описати як окремі функціональні підсистеми електронів металу, так і ефекти іх взаємного впливу з врахуванням багаточастинкових кореляційних ефектів.
} 